\title{
Mechanisms of Cognitive Impairment in Cerebral Small Vessel Disease: Multimodal MRI Results from the St George's Cognition and Neuroimaging in Stroke (SCANS) Study
}

\author{
Andrew J. Lawrence ${ }^{1 *}$, Bhavini Patel ${ }^{1}$, Robin G. Morris ${ }^{2}$, Andrew D. MacKinnon ${ }^{3}$, Philip M. Rich ${ }^{3}$, \\ Thomas R. Barrick', Hugh S. Markus ${ }^{1 *}$
}

1 Stroke and Dementia Research Centre, St George's University of London, London, United Kingdom, 2 Department of Psychology, Institute of Psychiatry, London, United Kingdom, 3 Department of Neuroradiology, Atkinson Morley Regional Neuroscience Centre, St George's Healthcare NHS Trust, London, United Kingdom

\begin{abstract}
Cerebral small vessel disease (SVD) is a common cause of vascular cognitive impairment. A number of disease features can be assessed on MRI including lacunar infarcts, T2 lesion volume, brain atrophy, and cerebral microbleeds. In addition, diffusion tensor imaging (DTI) is sensitive to disruption of white matter ultrastructure, and recently it has been suggested that additional information on the pattern of damage may be obtained from axial diffusivity, a proposed marker of axonal damage, and radial diffusivity, an indicator of demyelination. We determined the contribution of these whole brain MRI markers to cognitive impairment in SVD. Consecutive patients with lacunar stroke and confluent leukoaraiosis were recruited into the ongoing SCANS study of cognitive impairment in SVD $(n=115)$, and underwent neuropsychological assessment and multimodal MRI. SVD subjects displayed poor performance on tests of executive function and processing speed. In the SVD group brain volume was lower, white matter hyperintensity volume higher and all diffusion characteristics differed significantly from control subjects $(n=50)$. On multi-predictor analysis independent predictors of executive function in SVD were lacunar infarct count and diffusivity of normal appearing white matter on DTI. Independent predictors of processing speed were lacunar infarct count and brain atrophy. Radial diffusivity was a stronger DTI predictor than axial diffusivity, suggesting ischaemic demyelination, seen neuropathologically in SVD, may be an important predictor of cognitive impairment in SVD. Our study provides information on the mechanism of cognitive impairment in SVD.
\end{abstract}

Citation: Lawrence AJ, Patel B, Morris RG, MacKinnon AD, Rich PM, et al. (2013) Mechanisms of Cognitive Impairment in Cerebral Small Vessel Disease: Multimodal MRI Results from the St George's Cognition and Neuroimaging in Stroke (SCANS) Study. PLoS ONE 8(4): e61014. doi:10.1371/journal.pone.0061014

Editor: Jean-Claude Baron, University of Cambridge, United Kingdom

Received December 5, 2012; Accepted March 5, 2013; Published April 22, 2013

Copyright: (c) 2013 Lawrence et al. This is an open-access article distributed under the terms of the Creative Commons Attribution License, which permits unrestricted use, distribution, and reproduction in any medium, provided the original author and source are credited.

Funding: This research was funded by the Wellcome Trust (grant number 081589). The funder had no role in study design, data collection and analysis, decision to publish, or preparation of the manuscript.

Competing Interests: The authors have declared that no competing interests exist.

*E-mail: alawrenc@sgul.ac.uk (AJL); hmarkus@sgul.ac.uk (HSM)

\section{Introduction}

Cerebral small vessel disease (SVD) is the most common pathology underlying vascular dementia, and is a major cause of lesser degrees of vascular cognitive impairment (VCI) [1]. Radiological correlates are lacunar infarcts, with or without more diffuse areas of white matter hyperintensities $(\mathrm{WMH})$, also referred to as leukoaraiosis. Other features are brain atrophy and cerebral microbleeds. Cognitive impairment in SVD is characterised by prominent impairment of executive function and processing speed, with relative preservation of episodic memory [1,2]. Despite its importance, there are few specific treatments for cognitive impairment in SVD. The development of evidence based treatment approaches depends upon better understanding of the mechanisms of cognitive decline.

A number of potential mechanisms have been suggested, and their role investigated using magnetic resonance imaging (MRI). A popular hypothesis is that white matter damage causes white matter tract disruption and disconnection of cortical-subcortical and cortical-cortical connections underlying complex networks associated with cognitive control mechanisms and efficient information processing [3]. A number of pathologies seen in SVD could potentially cause such disruption including discrete lacunar infarcts, more diffuse regions of leukoaraiosis, and cerebral microbleeds (CMB).

MRI can be used to investigate the role of these different pathologies in causing cognitive impairment. In patients with SVD correlations between T2 lesion volume and cognition are weak [4,5]. This may reflect the fact that high signal on T2-weighted images represents increased water content and may not differentiate between areas of mildly and severely damaged tissue [6]. Diffusion tensor imaging (DTI) is more sensitive to white matter ultrastructural damage and DTI parameters have been shown to correlate more strongly with cognition than $\mathrm{T} 2$ lesion volume $[7,8,9]$. Not only can average diffusivity be measured as a marker of white matter ultrastructure, but the anisotropy of diffusion gives information on its directionality, and thus integrity of white matter structures. It has been recently suggested that additional information obtained from the diffusion tensor can give further insight in the nature of white matter damage. Axial and Radial components 
of the tensor have been proposed as markers with specificity to the type of neuronal damage [10]. Axial diffusivity (AD) represents diffusivity in the principal diffusion direction (i.e. in the gross orientation of white matter structure) and is a proposed marker of axonal damage, while radial diffusivity (RD), which is the average of diffusivities perpendicular to the principal direction of the tensor (and hence the gross orientation of white matter structure), is proposed to give information on the degree of demyelination. Such information may be relevant in SVD in which both ischaemic demyelination and axonal loss are seen pathologically [1].

Another feature associated with cognitive impairment in SVD is brain atrophy $[11,12]$. This could occur due to SVD pathology itself, or grey matter atrophy could occur secondary to white matter tract disruption producing denervation of cortical structures. Most studies in SVD have looked at global brain atrophy rather than differentiating grey and white matter volumes to assess whether changes in one particular brain compartment drive this association. CMB, small deposits of blood product detectable using blood sensitive MRI sequences such has T2*-weighted gradient echo, are also commonly observed in SVD [13] and have been linked to cognitive impairment [14].

Studies conducted to date have frequently looked at one MRI measure in isolation, and most have been in relatively small patient numbers. To investigate the role of these different pathologies, we applied multimodal MRI to a well phenotyped group of patients with SVD and correlated whole brain MRI measures with cognition. Whole brain measures were chosen as they are widely used [15], and technically suited to a role as a clinical marker of SVD where brain changes are anatomically diffuse and variable. We constructed multi-variable regression models to determine the strongest predictors of cognitive function. In addition, we extended upon previous investigations by assessing axial and radial diffusivity.

\section{Methods}

\section{Subjects}

(a)SVD patients. Consecutive patients with SVD were recruited to the St George's Cognition and Neuroimaging in Stroke (SCANS) study from stroke services in three hospitals covering a geographically contiguous area of South London (St George's Hospital, King's College Hospital and St Thomas's Hospital). SVD was defined as a clinical lacunar stroke syndrome [16] with an anatomically appropriate lacunar infarct on MRI, as well as confluent leukoaraiosis (Fazekas grade 2 or more) on MRI [17]. All patients were fluent in English to allow neuropsychological testing. Exclusion criteria were: any cause of stroke other than SVD including extra or intracranial arterial vessel stenosis $>50 \%$; any cardioembolic source; cortical infarcts; subcortical infarcts $>1.5 \mathrm{~cm}$ in diameter as these (striatocapsular type infarcts) are often due to embolism; other major central neurological system disorders; major psychiatric disorders (except depression); any cause for white matter disease other than SVD. Individuals with contraindications to MRI including claustrophobia were excluded.

The study was granted ethical approved by Wandsworth REC. 180 patients were screened of whom 137 volunteered to participate and gave written informed consent. 121 of the 137 SVD patients completed the protocol. Of non-completers, 6 withdrew due to the length of the neuropsychology examination, 2 could not complete MRI, 6 became unwell between consenting and testing, and 2 were found to meet exclusion criteria after consent, 1 due to narcolepsy and 1 due to schizophrenia.
All cognitive testing and MRI was performed at least 3 months post-stroke to minimise acute effects of stroke on cognition.

(b) Controls. For comparison with the SVD MRI data, a stroke-free control group was used. These were recruited as part of the St George's Neuropsychology and Imaging in Elderly (GENIE) study [18]. Briefly, individuals aged 50-90 at study onset were recruited by random sampling of family doctor lists; all individuals in the UK are registered with a family doctor. Screening ensured subjects were without central nervous system disease including stroke and transient ischaemic attack (TIA). As GENIE is a population study investigating age related change in cognition and MRI, no screening was conducted based on MRI data. MRI scans performed at the 2 year follow-up point were used as these were obtained using the same MRI scanner and sequences used for the SVD group. At this time point MRI data was available for 57 subjects.

\section{Clinical assessments}

All SVD patients were examined by a neurologist. Cerebrovascular risk factors recorded including hypertension, diabetes mellitus, hypercholesterolaemia, body mass index and smoking history as well as current medications. Hypertension was defined as either systolic $\mathrm{BP}$ of $>140 \mathrm{mmHg}$, diastolic $\mathrm{BP}>90 \mathrm{mmHg}$ or on treatment. Hypercholesterolaemia was defined as a random total cholesterol of $>5.2 \mathrm{mmol} / \mathrm{L}$ or on treatment. Smoking was divided into current, ex-smoker and never. Diabetes mellitus was defined as being on drug or insulin treatment. Disability was assessed using the modified Rankin scale [19].

\section{MRI Acquisition}

Images were acquired using a 1.5-T General Electric Signa HDxt MRI system (General Electric, Milwaukee, WI, USA) with a maximum gradient amplitude of $33 \mathrm{mTm}^{-1}$ and a proprietary head coil. Sequences were acquired across the whole brain and total imaging time was approximately 45 minutes. All subjects were placed in the head coil in a neutral position with an alignment marker at the nasal bridge to standardise head position. Minimal head movement was ensured during the scan by use of foam pads and a Velcro strap.

\section{Conventional Imaging}

(1) Axial Fluid Attenuated Inversion Recovery (FLAIR) sequence-TR/TE/TI $=9000 / 130 / 2200 \mathrm{~ms}$, field of view $($ FOV $)=240 \times 240 \mathrm{~mm}^{2}$, matrix $=256 \times 192,28$ contiguous slices of $5 \mathrm{~mm}$ thickness.

(2) Coronal spoiled gradient recalled echo T1-weighted (SPGR) sequence-TR $/ \mathrm{TE}=11.5 / 5 \mathrm{~ms}, \mathrm{FOV}=240 \times 240 \mathrm{~mm}^{2}$, matrix $=256 \times 192$, Flip Angle $=18^{\circ}, 176$ contiguous slices of $1.1 \mathrm{~mm}$ thickness.

(3) Gradient Recalled Echo (GRE) T2*-weighted sequence-TR/ $\mathrm{TE}=300 / 30 \mathrm{~ms}, \mathrm{FOV}=240 \times 240 \mathrm{~mm}^{2}$, matrix $=256 \times 192$, 28 axial slices of $5 \mathrm{~mm}$ thickness with no slice gap. Imaging sequences were identical in cases and controls but T2-GRE sequences were not obtained for control subjects.

\section{Diffusion Tensor Imaging}

Axial single shot spin echo planar images $(\mathrm{EPI}$; TE $=93.4 \mathrm{~ms}$, $\mathrm{TR}=15600 \mathrm{~ms}$ ) were acquired to give isotropic voxels and whole brain coverage $\left(2.5 \mathrm{~mm}^{3} ; \mathrm{FOV}=240 \times 240 \mathrm{~mm}^{2}\right.$, acquisition matrix $=96 \times 96$ ). Following four acquisitions without diffusion weighting $\left(b=0 \mathrm{~s} \mathrm{~mm}^{-2}\right)$, diffusion sensitised images were acquired with gradients applied $\left(b=1000 \mathrm{~s} \mathrm{~mm}^{-2}\right)$ in 25 non- 
collinear directions. This was repeated to acquire a further four $\mathrm{b}=0 \mathrm{~s} \mathrm{~mm}^{-2}$ images and the negative of the 25 directions. Images were realigned to remove eddy current distortions using the FSL Linear Image Registration Tool (FLIRT, FMRI Software Library, FSL version 4.1; FMRIB Analysis Group, Oxford, UK, http:// www.fmrib.ox.ac.uk/fsl [20]). The geometric average of the positive and negative acquisitions was taken to eliminate gradient cross-terms [21]. The eight $b=0 \mathrm{~s} \mathrm{~mm}^{-2}$ images were coregistered and averaged to give a T2-weighted EPI image (henceforth termed the b0).

\section{MRI Data analysis}

MRI scans were analysed blinded to subject identity.

WMH Lesions. White matter hyperintense regions of FLAIR images were delineated by a single rater, using the semi-automated DISPUNC program [22] (David Plummer, University College, London, UK). Within each slice, for each lesion, the user identifies a voxel near the edge of the lesion and an intensity-gradient-based algorithm uses this starting point to automatically delineate the contour of lesion extent. Lesions that were $\geq 2 \mathrm{~mm}$ in diameter were delineated. Whole brain lesion maps were generated and lesion load was then calculated as the percentage of nonnormalised brain volume. Brain volume computation techniques are presented below.

Lacunar infarcts. A single consultant neuroradiologist evaluated the T1-weighted and FLAIR images for lacunar infarcts, defined as a CSF filled cavity within the white matter or subcortical regions, between $3-15 \mathrm{~mm}$ in diameter.

Gerebral Microbleeds. Microbleeds were identified on GRE as well-defined focal areas of low signal $<10 \mathrm{~mm}$ in diameter. Symmetrical areas of basal ganglia calcification, flow voids from blood vessels and low signal due to adjacent bone were discounted. The Brain Observer Microbleed Rating Scale (BOMBS) was used to describe the location of microbleeds [23]. Only microbleeds meeting the "certain" criteria were analysed for this study.

Normalised Brain Volume. Normalised Brain Volume (NBV) is an estimate of brain size with respect to skull size and thus a measure of brain atrophy in cross-sectional data [24]. T1weighted structural images were segmented into tissue probability maps for Grey Matter (GM), White Matter (WM) and CerebroSpinal Fluid (CSF) components using the segment function of the Statistical Parametric Mapping (SPM) package (version 8; Wellcome Department of Cognitive Neurology, London; http://www. fil.ion.ucl.ac.uk/spm). GM and WM tissue maps were of the whole brain, with the GM map including both cortical and subcortical tissue. NBV and non-normalised brain volume were then calculated using the automated SIENAX program (FSL version 4.1) [24]. To minimise tissue misclassification due to the presence of lesions, WMH lesion maps were used to correct GM and WM volumes. The correction was carried out by calculating the volume of tissue classified as GM within the WMH region. This figure was then subtracted from the GM volume total and added to the WM volume total. This ensured that hypointense T1-weighted signal in the WM attributable to leukoaraiosis was not misclassified as GM for volume analysis. All tissue segmentations were visually inspected to ensure consistent segmentation results.

Diffusion Tensor Histogram Analysis. DTIs were calculated at each voxel from diffusion-weighted images using the loglinear fitting method of Basser et al [25] and Fractional Anisotropy (FA), Mean Diffusivity (MD), AD and RD maps were computed.

To identify normal appearing white matter (NAWM) tissue in DTI space, multimodal coregistration was performed using FLIRT. T1-weighted structural images and T2-weighted FLAIR images were registered to the b0 image using 12 parameter affine registration with a normalised mutual information cost function. These transformations were applied to the tissue probability maps and WMH lesion masks. NAWM in the DTIs was classified as non-lesion voxels where the probability of WM was greater than GM or CSF.

Histograms were calculated for FA, MD, AD and RD maps for NAWM voxels. Each histogram had 100 bins: FA bin width: 0.01 , range: 0 to $1 . \mathrm{MD}, \mathrm{AD}$ and $\mathrm{RD}$ bin widths: $4 \times 10^{-5} \mathrm{~mm}^{2} \mathrm{~s}^{-1}$, range: 0 to $4 \times 10^{-3} \mathrm{~mm}^{2} \mathrm{~s}^{-1}$. Histogram frequencies were normalised by the total NAWM to correct for individual differences in NAWM volume.

Distributions of FA and diffusivity measures in NAWM are nonGaussian. For this reason analysis is usually conducted on measures derived from the histogram, therefore histogram measures of Normalised Peak Height (NPH), and Peak Value (or peak location) are preferable to means and standard deviations [8]. Peak values are where DTI measures exhibit maximum frequency. NPH is the proportion of observations at the peak value. We also calculated measures of centrality (mean, median), variability (standard deviation, interquartile range) and distribution shape (skew, kurtosis).

Missing MRI Data. Complete MRI scanning data was unavailable in 5 SVD subjects: In four cases excessive geometrical EPI warping was observed in diffusion-weighted images causing co-registration errors. One SVD subject was unable to complete MRI. Complete scanning data was not available for 5 control cases. Of these, two had FLAIR scans which were technically inadequate for WMH lesion volume measurement (excessive movement; acquisition error), one was unable to complete MRI, and two exhibited excessive EPI warping. Consequently complete MRI data were acquired for 115 SVD patients and 50 controls.

\section{Cognitive Function Assessment}

In the SVD group cognitive assessment was performed by a neuropsychologist using a battery of widely-used tasks chosen to characterise the cognitive impairment seen in SVD. Assessment was within 2 weeks of MRI, taking approximately 2.5 hours. Index measures summarising performance across related tasks were produced as detailed in Table S1 in File S1. In addition a Global Cognition index was produced which summarised performance on all the tasks. Premorbid IQ was estimated using the restandardised National Adult Reading Test (NART; $2^{\text {nd }}$ Edition) [26].

Cognitive Index Construction. To construct each index, key measures for each task were transformed into z-scores through psychometric standardisation using the best available age-scaled normative data. An exception was the version of the modified Wisconsin Card Sort Test (mWCST), selected for brevity and sensitivity, for which a published study was used to select normative data with comparable age and gender distribution [27]. Task z-scores were averaged to create the cognitive index scores, obtaining reliable indices (see Results section)

Missing Cognitive Data. For missing data where the subject was unable to complete the task, the minimum scaled score attainable for that task was substituted (this was 0 or 1 ; corresponding to $\mathrm{z}$-scores of -3.33 and -3 ). If data were missing for other reasons, the cognitive index scores were calculated from the remaining tasks in the index to reduce the cumulative impact of sporadic data loss on sample size. Such missing data comprised $1.2 \%$. 


\section{Statistical Analysis}

Data analysis was conducted using R [28]. MRI data were compared between SVD and control groups using t-tests. Repeated measures ANOVA was conducted treating the 6 cognitive indices as a factor to confirm that performance was significantly different across cognitive domains. Custom linear contrasts assessed the a-priori hypothesis that executive function and processing speed would be the most impaired [2,29], and would therefore show deviation scores significantly different from each of the other 4 indices. One-sample t-tests tested the nullhypothesis that each cognitive index was equal to zero-i.e. that the average performance in the group was equal to the expected performance of age-matched controls.

Within the SVD group, we examined associations between MRI parameters and the key cognitive measures that showed profile based impairment, namely Executive Function and Processing Speed (see Results section). Multiple regression models were used, adjusting for confounder variables: age, gender \& premorbid IQ (NART). First, models were constructed for each MRI variable controlling for confounders: "single predictor" models. Second, models including the confounders and all MRI variables were constructed to assess the relative importance of correlated predictors, these are termed "multi-predictor" models. For all models standardised regression coefficients were used to assess relative importance of predictors. Regression results were visually screened for outliers, checked for variance inflation of regression coefficients due to multicollinearity and the quality of overall fit tested with F-tests. Measures of multicollinearity are high when correlated predictors derived from directly related measures are included together in multi-variable models [30]. This is the case for (a) FA, MD, AD and RD histograms, (b) normalised GM, WM and total brain volumes, (c) CMB count and CMB presence variables. To avoid multicollinearity of predictors in the regression model, for each of the three sets above, only the measure with the strongest univariate association was included in the multi-predictor analysis (see Results).

\section{Results}

Final subject numbers for analysis were 115 (SVD) and 50 (Controls). A summary of demographic data for patients and controls is displayed in Table 1 .
Table 2. MRI results in SVD cases and controls.

\begin{tabular}{|c|c|c|c|}
\hline MRI Measure & SVD & Controls & $p$ value \\
\hline NBV (whole brain, ml) & $1295.1(91.1)$ & $1337.3(87.3)$ & $\mathrm{p}<0.005$ \\
\hline Grey matter normalised vol. (ml) & $727.9(75.4)$ & $792.3(62.2)$ & $\mathrm{p}<0.0001$ \\
\hline White matter normalised vol. (ml) & $567.1(71.9)$ & $544.9(52.0)$ & $\mathrm{p}<0.043$ \\
\hline WMH lesion vol. (ml) & $31.87(26.97)$ & $8.7(11.73)$ & $\mathrm{p}<0.0001$ \\
\hline WMH lesion load (\% brain) & $3.19(2.64)$ & $0.84(1.12)$ & $\mathrm{p}<0.0001$ \\
\hline Lacune Count & $4.26(5.48)$ & $0.65(1.51)$ & $\mathrm{p}<0.0001$ \\
\hline $\begin{array}{l}\text { Lacune Count Quartile } \\
\text { Descriptives }\end{array}$ & $0,1,2,5,27$ & $0,0,0,1,10$ & - \\
\hline CMB Count & $4.73(16.64)$ & - & - \\
\hline CMB Count Quartile Descriptives & $0,0,0,2,144$ & - & - \\
\hline
\end{tabular}

Values: Mean (Standard Deviation). Quartile descriptives: Minimum, lower quartile, median, upper quartile, maximum; NBV-Normalised Brain Volume, WMH-White Matter Hyperintensity.CMB-Cerebral Microbleed.

doi:10.1371/journal.pone.0061014.t002

\section{MRI}

(a) Conventional MRI parameters. Comparisons of MRI parameters between SVD cases and controls are shown in Table 2. NBV was lower in SVD compared to controls $(p<0.0001)$, and this difference appeared to be driven by a reduction in grey matter volumes, which were also significantly lower in the SVD group. WMH lesion load was higher in SVD compared to controls $(3.19 \mathrm{v} 0.84 \%, \mathrm{p}<0.0001)$ as was the number of Lacunar infarcts $(\mathrm{p}<0.0001)$.

Distributions of lacunar infarct count and CMB count were skewed (Table 2, quartile descriptives), particularly for microbleeds, with most subjects $(60 \%)$ having no detectable microbleeds. Accordingly these variables were $\log 10$ transformed to reduce skew, and for microbleeds the presence of microbleeds was used as a grouping variable to compare those with microbleeds to those without in addition to using the $\log 10$ number of microbleeds as a continuous predictor.

(b) DTI. Histograms of FA, MD, $\mathrm{AD}$ and $\mathrm{RD}$ values in NAWM are displayed in Figure 1. Histogram analysis revealed significant differences between SVD and control groups (Table 3).

1) FA was lower in SVD cases compared with controls. The shape of the distribution differed with increased skew and

Table 1. Demographics of SVD and control groups.

\begin{tabular}{llll}
\hline & & & \\
\hline & SVD Patients (N= 121) & Controls (N= 57) & Group Test Result \\
\hline Age (years) & $70.01(9.75)$ & $70.36(9.18)$ & $\mathrm{p}<0.82$ \\
Gender (\% male) & $78(64.5)$ & $35(62.4)$ & $\mathrm{p}<0.25$ \\
Hypertension (\%) & $112(92.6)$ & $28(49.1)$ & $\mathrm{p}<0.0001$ \\
Systolic BP (mmHg) & $146.8(21.47)$ & $138.48(18.04)$ & $\mathrm{p}<0.013$ \\
Diastolic BP (mmHg) & $80.95(10.77)$ & $79.27(12.33)$ & $\mathrm{p}<0.36$ \\
Cholesterol (mmol) & $4.333(0.899)$ & $5.67(1.13)$ & $\mathrm{p}<0.0001$ \\
Diabetes Mellitus (\%) & $24(19.8)$ & $0(0)$ & $\mathrm{p}<0.0001$ \\
$\begin{array}{l}\text { Smoker (current or ex) } \\
\text { BMI (kg m }{ }^{-2} \text { ) }\end{array}$ & $55(45.5)$ & $32(56.1)$ & $\mathrm{p}<0.185$ \\
\hline $\begin{array}{l}\text { BP-Blood Pressure; DM-Diabetes Mellitus; BMI-Body Mass Index. All values are Mean (SD) or proportion (\%) as specified. Independent sample t-test or Chi-squared analysis } \\
\text { used as appropriate. } \\
\text { doi:10.1371/journal.pone.0061014.t001 }\end{array}$ & $27.05(4.88)$ & & $\mathrm{p}<0.016$ \\
\end{tabular}


kurtosis in SVD. Variability of FA values about the mean was comparable: Standard Deviation (SD), peak height and inter-quartile range (IQR) did not differ between groups.

2) MD was higher in SVD cases compared with controls. MD values were also more variable in SVD cases (as shown by $\mathrm{SD}$, IQR, peak height). In contrast to FA histograms, MD histograms were less skewed and less leptokurtic in the SVD group.

3) Differences between SVD cases and controls were greater for $\mathrm{RD}$ measures than for $\mathrm{AD}$ measures. The pattern of change seen for RD was similar to that for MD.

4) DTI measures were highly correlated, particularly MD which is arithmetically derivable from the $\mathrm{AD}$ and $\mathrm{RD}$ measures. Correlation coefficients for median diffusion tensor parameters in NAWM are displayed in Table S2 in File S1

\section{Cognitive Results}

Cognitive Index Consistency. Cronbach's Alpha for cognitive indices ranged from 0.69 (Processing Speed) to 0.85 (both Verbal and Performance Intelligence), indicating good internal consistency (see Table S3 in File S1). The Global Cognition index had an alpha of 0.91 indicating very high consistency of general performance across the task battery.

Gognitive Profile. The profile of cognitive performance in the SVD subjects is shown in Figure 2. There was a significant within-subject effect of cognitive domain $(\mathrm{p}<0.001)$. Performance on Executive Function and Processing Speed was poorer than performance on Working Memory $(\mathrm{p}<0.001)$, Long-Term Memory $(p<0.001)$, Performance Intelligence $(p<0.001)$ and Verbal Intelligence $(\mathrm{p}<0.001)$. No such difference was detectable between Executive Function and Processing Speed $(\mathrm{p}<0.99)$. Executive Function and Processing Speed are the cognitive indices primarily

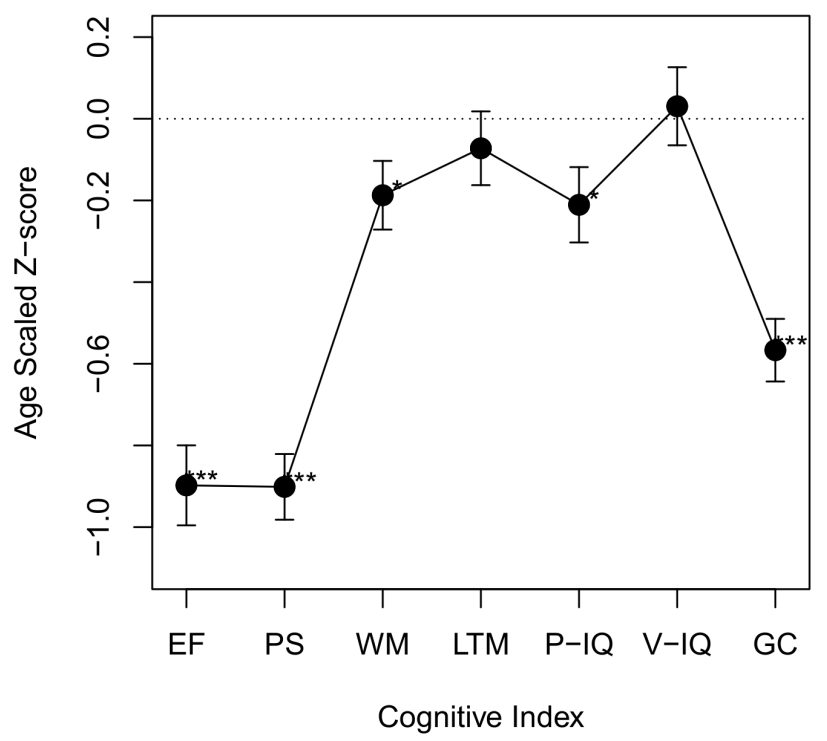

Figure 1. DTI histograms of Normal Appearing WM in SVD and control groups. Normalised frequency histograms of diffusion tensor imaging data are presented for SVD (solid lines) and control (dashed lines) groups. FA-Fractional Anisotropy; MD-Mean Diffusivity $\left(\times 10^{-3} \mathrm{~mm}^{2} \mathrm{~s}^{-1}\right)$; AD-Axial Diffusivity $\left(\times 10^{-3} \mathrm{~mm}^{2} \mathrm{~s}^{-1}\right)$; Radial Diffusivity $\left(\times 10^{-3} \mathrm{~mm}^{2} \mathrm{~s}^{-1}\right)$. SVD-Small Vessel Disease Group. CON-Control Group.

doi:10.1371/journal.pone.0061014.g001
Table 3. DTI Histogram Group Results.

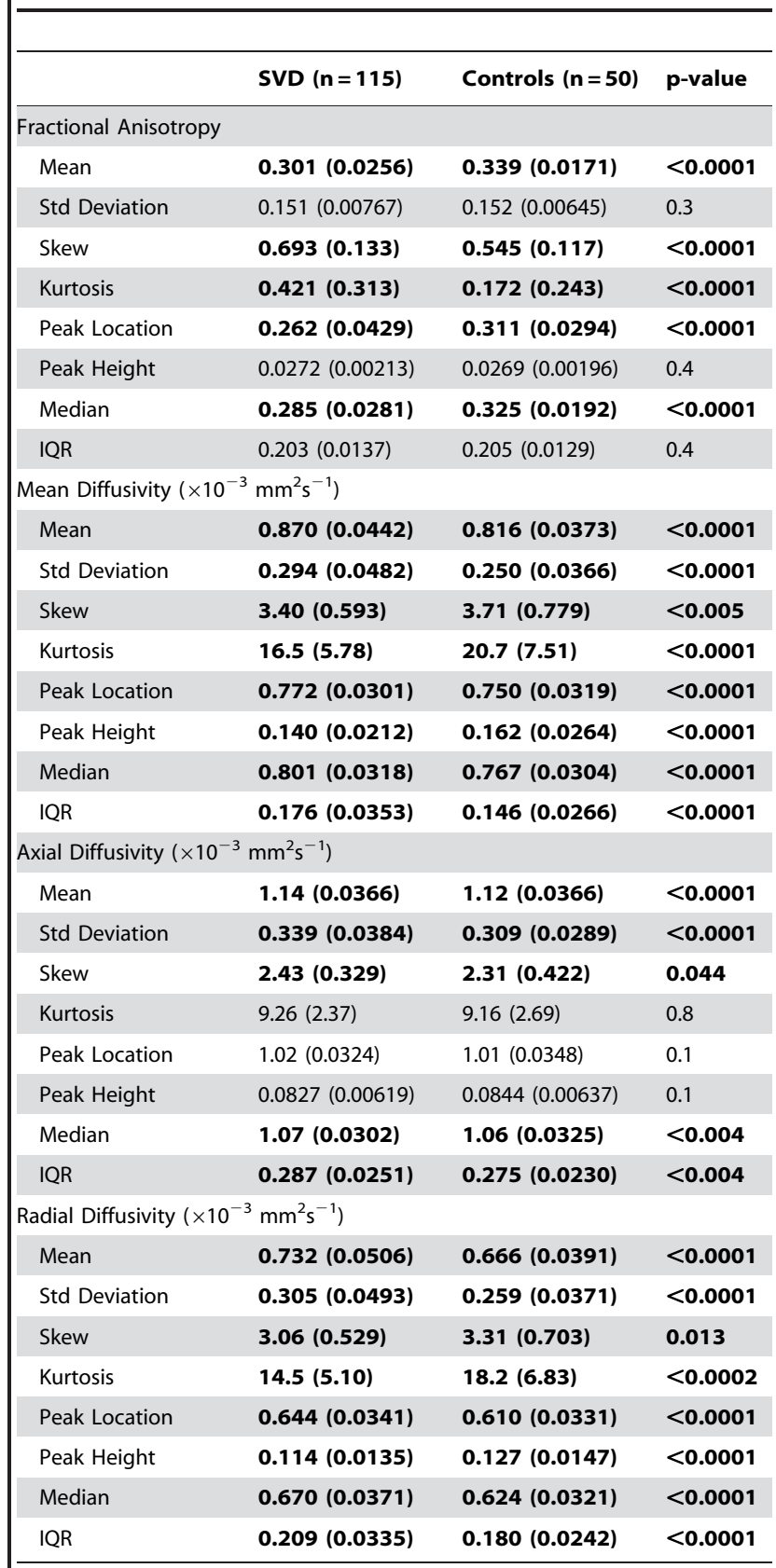

Std. Deviation-Standard Deviation; Peak Location-mode of the histogram; Peak Height-normalised frequency of the mode. Test results in bold are significant at $p<0.05$ level (uncorrected).

doi:10.1371/journal.pone.0061014.t003

impaired in SVD (Pantoni 2010, Zhou \& Jia 2009). In our SVD group Working Memory and Performance IQ also significantly differed from expected performance, however, the magnitude of this difference was significantly lower than for Executive Function and Processing Speed. To focus our analysis on the significant determinants of cognitive impairment in SVD we restrict further analysis to the Executive Function and Processing Speed indices.

\section{Predictors of Executive Function \& Processing Speed}

(a) Single Predictor Models. Regression coefficients between MRI variables and the Executive Function and Processing 

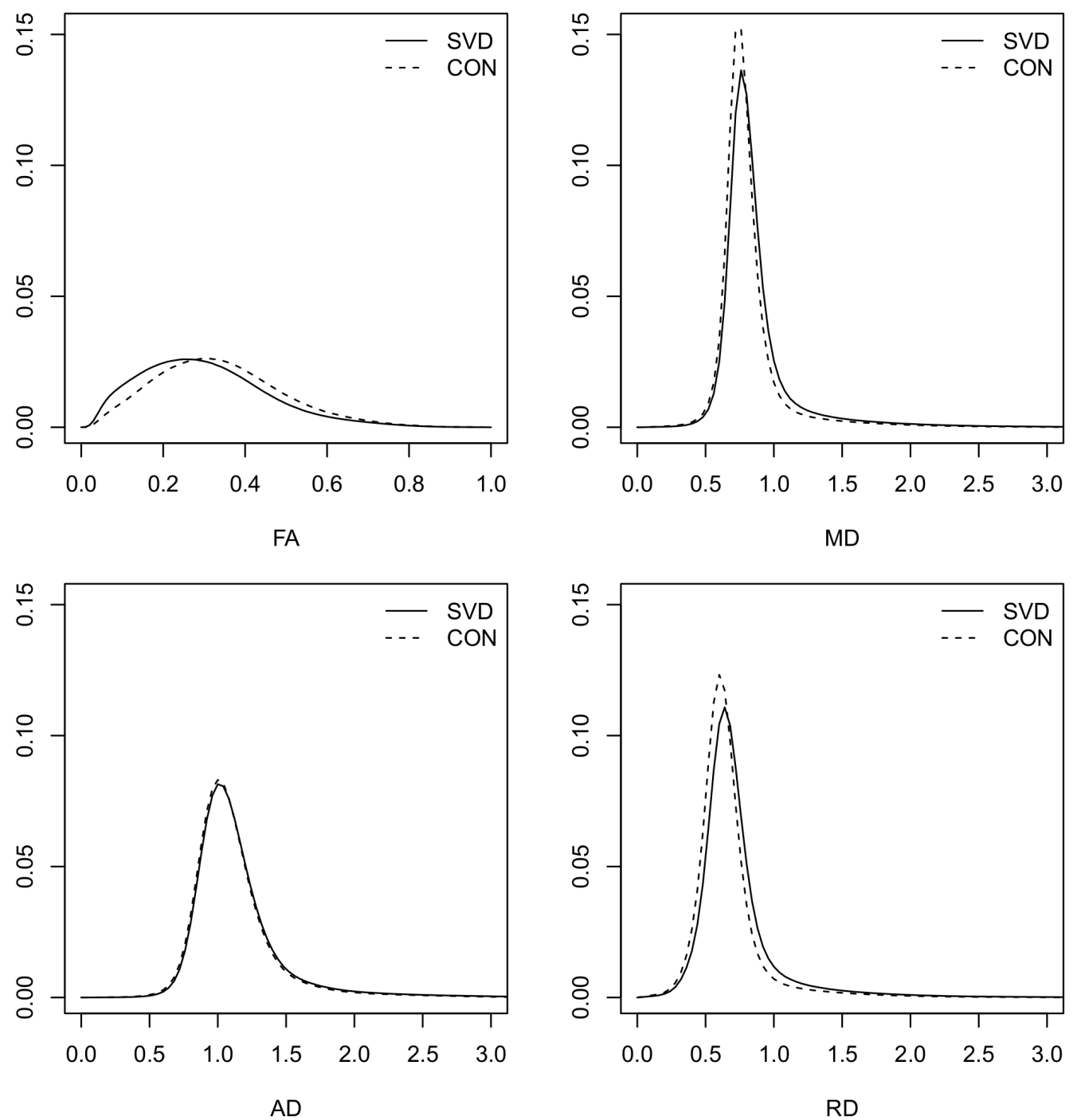

Figure 2. Cognitive Profile in the SVD Group. Average scores for cognitive indices are presented for the SVD group. Error Bars represent $+/-1$ standard error of the mean. Solid line at zero represents the expected performance for a group of average ability. EF-Executive Function; PSProcessing Speed; WM-Working Memory; LTM-Long-term "episodic" memory; PIQ-Performance IQ; VIQ-Verbal IQ; GC-Global Cognition measure. ***index score significantly different from zero, $\mathrm{p}<0.001$; *index score significantly different from zero, $\mathrm{p}<0.05$. doi:10.1371/journal.pone.0061014.g002

Speed cognitive measures are shown in Table 4. The pattern of associations with MRI variables was similar for the two cognitive measures. Both NBV and lacunar infarct count were significantly related, along with the volume of grey matter, but not white matter. Lower executive function and processing speed scores were associated with reduced normalised volume (i.e. greater atrophy) and greater numbers of lacunes. Relationships between DTI histogram measures and cognition are shown for FA, MD, AD and RD; all exhibited significant associations. Associations were stronger and more consistent for MD and RD measures, and weaker and less consistent for $\mathrm{AD}$, which did not survive multiple comparisons correction. Overall the strongest association for a diffusion measure was found for the normalised peak height of the RD histogram for Executive Function, and the equivalent MD measure for Processing Speed. In contrast WMH lesion load showed a weak association with Processing Speed which did not survive multiple comparisons correction, and no significant association with Executive Function. Furthermore, neither the number of, nor presence of, microbleeds showed a significant association with the key cognitive measures after correction for multiple comparisons.

(b) Multi-predictor Models. Multiple regression was performed to evaluate the relative contribution of the different MRI measures. For highly correlated MRI measures (see statistical methods section), the most significant univariate predictor was included in the multi-predictor model. For Executive Function these were total NBV, CMB count and the normalised peak height of the RD histogram. For Processing Speed, these were total NBV, CMB count and the normalised peak height of the MD histogram. As MD and RD and highly correlated, the above analyses were repeated using MD normalised peak height for Executive Function and RD normalised peak height for Processing Speed. Calculated coefficients were similar (and were identical to 2 decimal places) leading to identical model interpretation.

The regression model (Table 4) provided a highly significant fit for the Executive Function index; the proportion of variance explained was $0.6(\mathrm{~F}=19.8, \mathrm{p}<0.00001)$. The strongest MRI predictor of the Executive Function index was Lacunar infarct 
Table 4. Predictors of key cognitive domains in SVD.

\begin{tabular}{|c|c|c|c|c|}
\hline \multirow[b]{2}{*}{ Predictors } & \multicolumn{2}{|c|}{ Single-predictor Models ${ }^{1}$} & \multicolumn{2}{|c|}{ Multi-predictor Models ${ }^{2}$} \\
\hline & EF & PS & EF & PS \\
\hline NBV (Whole brain) & $.281(.0001)$ & $.387(<.0001)$ & $.119(.25)$ & $.270(.0026)$ \\
\hline Normalised GM vol. & $.183(.011)$ & $.313(.0001)$ & - & - \\
\hline Normalised WM vol. & $.152(.046)$ & $.140(.11)$ & - & - \\
\hline WMH lesion load (log, \%) & $-.080(.25)$ & $-.200(.013)$ & $.143(.21)$ & $-.058(.54)$ \\
\hline Lacunar Infarct Count (log) & $-.286(<.0001)$ & $-.355(<.0001)$ & $-.227(.019)$ & $-.244(.006)$ \\
\hline \multicolumn{5}{|l|}{ Cerebral Microbleeds } \\
\hline Number (log) & $-.156(.026)$ & $-.108(.21)$ & $-.038(.6)$ & $.113(.22)$ \\
\hline Presence (Group) & $-.042(.5)$ & $-.059(.5)$ & - & - \\
\hline \multicolumn{5}{|l|}{ Fractional Anisotropy } \\
\hline Histogram Peak Location & $.224(.002)$ & $.268(.0008)$ & - & - \\
\hline Histogram Peak Height & $-.105(.14)$ & $-.215(.007)$ & - & - \\
\hline \multicolumn{5}{|l|}{ Mean Diffusivity $\left(\mathrm{mm}^{2} \mathrm{~s}^{-1}\right)$} \\
\hline Histogram Peak Location & $-.150(.035)$ & $-.175(.031)$ & - & - \\
\hline Histogram Peak Height & $.263(.0002)$ & $.281(.0008)$ & - & $.085(0.41)$ \\
\hline \multicolumn{5}{|l|}{ Axial Diffusivity $\left(\mathrm{mm}^{2} \mathrm{~s}^{-1}\right)$} \\
\hline Histogram Peak Location & $-.067(.4)$ & $-.088(.29)$ & - & - \\
\hline Histogram Peak Height & $.184(.010)$ & $.181(.030)$ & - & - \\
\hline \multicolumn{5}{|l|}{ Radial Diffusivity $\left(\mathrm{mm}^{2} \mathrm{~s}^{-1}\right)$} \\
\hline Histogram Peak Location & $-.175(.015)$ & $-.204(.012)$ & - & - \\
\hline Histogram Peak Height & $.278(.0002)$ & $.243(.005)$ & $.211(.046)$ &.- \\
\hline
\end{tabular}

Values show Standardised regression coefficients: $\beta$ ( $p$-value) for predictor variables in regression models of: EF-Executive Function, and PS-Processing Speed.

${ }^{1}$ Single variable models control for effects of age, gender and NART-IQ. Values in Bold remain significant after multiple comparisons correction (Holm-Bonferroni).

${ }^{2}$ Multiple variable models include all the terms indicated as well as age, gender and NART-IQ. A subset of variables were included in the multi-predictor models, as described in the statistical analysis part of the methods section. Models were highly significant: $E F: R^{2}=0.60 ; F_{(8,105)}=19.8, p<0.0001 ; P S: R^{2}=0.49 ; F_{(8,105)}=12.8, p<0.0001$. Significant terms in each model are highlighted in bold.

doi:10.1371/journal.pone.0061014.t004

count $(\beta=-0.227)$ followed by the DTI measure: normalised peak height of the white matter radial diffusivity histogram $(\beta=0.211)$. The other predictors in the model: NBV, WM lesion load and the presence of microbleeds did not reach significance. Similar results were obtained for the Processing Speed index $\left(\mathrm{R}^{2}=0.5, \mathrm{~F}=12.8\right.$, $\mathrm{p}<0.0001)$, although NBV was now a significant predictor $(\beta=-0.270)$ and the DTI measure was not $(\beta=0.085)$. Post-hoc t-tests based on standardised regression coefficients and pooled variance (analogous to the Hotelling/Williams test for dependent correlation coefficients) were conducted to look for significant differences in the regression coefficients obtained for each predictor in the two cognitive models, none reached significance.

\section{Discussion}

In this well phenotyped cohort of patients with symptomatic small vessel disease and radiological leukoaraiosis, we found a characteristic cognitive profile with impaired performance in executive function and processing speed, relative to working memory and episodic memory. Using a multimodal MRI approach, we investigated the mechanisms underlying this cognitive impairment. On analysis of single predictors, impaired executive function and processing speed were associated with lacunar infarct count, reduced brain volume, and white matter ultrastructural damage determined using DTI. Multi-predictor analysis showed that $60 \%$ of variance in executive function and $50 \%$ of variance in processing speed was explained by the regression model. For executive function, the strongest predictor was lacunar infarct count followed by the DTI measure. Normalised brain volume and lacunar infarct count were the predictors of processing speed. In these multivariable analyses $\mathrm{WMH}$ lesion load, and the presence or number of microbleeds, were not independently associated

Most, but not all, previous studies in sporadic and hereditary (CADASIL) SVD, have found an association between lacunar infarcts and cognition. The independent associations we found with lacunar infarct count may well be mediated by infarcts leading to white matter tract disruption and subsequent corticalsubcortical disconnection. Recent studies using higher magnetic field MRI have shown micro infarcts within the cortex and it is possible that these infarcts, if they associate with total lacunar infarct count, may contribute to this relationship with cognitive impairment [31].

The independent association found for DTI measures supports the role of white matter ultrastructural damage in causing executive dysfunction. Consistent with previous studies $[7,8]$, including the results of the large multi-centre LADIS study [9,32], our results show that DTI measures are more sensitive to white matter damage than WMH lesion load. We evaluated a number of markers of the DTI histogram. Associations were generally stronger and more consistent for MD than for FA.

It has been suggested that axial and radial components of the DTI tensor may give further information about the nature of neuronal damage [33] although this has not been previously studied in SVD. AD may represent a marker of axonal damage 
while RD has been proposed as a marker of demyelination [10]. Both ischaemic demyelination and axonal damage have been reported in neuropathological studies of SVD [34]. Both AD and $\mathrm{RD}$ were abnormal in the SVD group relative to controls, but RD was the stronger predictor of executive dysfunction. This may reflect the importance of ischaemic demyelination vs. axonal degeneration in determining the presence of cognitive impairment in SVD, alternatively it may represent relatively greater reliability of the second and third eigenvalues (RD) relative to the first (AD). This analysis has two implications. First, in studies monitoring white matter damage in SVD, RD is a better marker than AD. Second, it provides some support for the role of demyelination in SVD-related cognitive dysfunction.

Previous studies in both sporadic and genetic SVD have reported associations between brain volume and cognition, and in those studies where multimodal MRI has been performed, this association has tended to be independent of the effects of other MRI variables [15,35]. In this study we found reduced whole brain volume, which was attributable to lower grey matter volume, rather than a reduction in white matter volume. Overall, whole brain volume acted as a better single predictor of cognitive performance than grey matter volume, and in the multi-predictor model a strong independent association was observed between brain volume and processing speed, but this did not reach statistical significance for executive functioning. Although counterintuitive, our finding of WM volume in the high normal range is not necessarily evidence against a key role for demyelination in SVD. While loss of myelin would be expected with volume loss, other white matter pathological processes in SVD including oedema and inflammation, might be associated with volume increase. Of relevance, investigations of $\mathrm{WM}$ volume in multiple sclerosis have produced inconsistent findings [36], and this has been explained by the presence of opposing processes (inflammation, gliosis, oedema) acting to increase observed WM volume and counteract the effects of demyelination on WM volume [36,37].

We found no association between the presence or number of microbleeds and cognitive function. Previous results have produced different conclusions as to the relationship of microbleeds to cognitive dysfunction in SVD $[14,13]$. The distribution of microbleeds was skewed with a large number of microbleeds in a number of patients. In a post-hoc analysis [38] we found that a high microbleed count (8 or greater, representing the top $10 \%$ of the sample) was related to executive function. However, even the contribution of high levels of microbleeds appears to explain only a small amount of the variation in cognitive function.

\section{Limitations}

The term SVD is used to describe a variety of clinical presentations ranging from asymptomatic white matter hyperintensities in community studies, through to multiple lacunar infarcts and leukoaraiosis in patients with vascular dementia. Furthermore, in patients with symptomatic lacunar stroke due to SVD, it has been suggested there may be more than one underlying pathology. The role of atherosclerosis has been proposed in patients with isolated lacunar infarcts without leukoaraiosis, while a diffuse arteriopathy has been proposed in patients with multiple smaller lacunar infarcts and leukoaraiosis $[39,40]$. It is possible the mechanisms causing cognitive impairment in these different situations vary. For this reason we recruited a homogenous group of patients who all presented with symptomatic lacunar infarction and had confluent leukoaraiosis on MRI. This would match with the proposed subtype characterised as secondary to a diffuse arteriopathy.

Axial and radial diffusivity measures, unlike FA and $\mathrm{MD}$, are dependent on eigenvalue sorting and this effect is greater in complex brain tissue such as regions of crossing fibres and pathology [41]. This has implications in interpretation of findings as caution is required in linking $\mathrm{AD}$ and $\mathrm{RD}$ measures to axonal and demyelinating pathologies in white matter [41]. White matter regions in SVD patients did not show the pattern of changes in $\mathrm{AD}$ described as characteristic of axonal damage. Relative to controls, $\mathrm{AD}$ was increased, not decreased and this may reflect different disease processes such as tissue oedema, masking the effects of axonal degeneration. Furthermore, MD and RD measures were highly correlated in this sample, and produced interchangeable results in the multivariable models. Although here we address the question of $\mathrm{RD}$ and $\mathrm{AD}$ as pathological markers separately to the question of the value of conventional $\mathrm{MD}$ and FA measures, the extent to which RD and MD provide meaningfully different information about the underlying tissue ultrastructure is unclear. However the results from this baseline analysis suggest they may provide similar information, at least in SVD. This, together with concerns about the validity of the $\mathrm{AD}$-axonal degeneration, and the RD-demyelination link in complex or lesioned WM, suggests further research is required to fully elucidate the relationship between neuropathology and these inter-correlated measurements in SVD.

The whole brain MRI measures presented in this analysis are commonly used [15], and are suited to identifying anatomically diffuse and variable SVD brain changes, however there is mounting evidence that the location of damage in SVD may be important $[42,43,44]$, and provide further insight to our understanding of mechanisms in SVD.

Further longitudinal studies correlating MRI changes with cognition are now required. The SCANS dataset described in this paper is the baseline analysis from such a study which is currently performing yearly MRI and cognition over a period of four years.

\section{Supporting Information}

File S1 Supplementary Results Tables. Table S1, Cognitive indices and task measures. Table S2, Inter-individual Median Diffusion Measure Correlations. Table S3, Internal Reliability of Cognitive Indices.

(DOG)

\section{Acknowledgments}

Recruitment to the study was supported by the English National Institute of Health Research Clinical Stroke Research Network. We thank Lalit Kalra and Tony Rudd for help with recruitment. We also thank Thomas Willis for assistance with neuropsychological assessment and Ai Wern Chung for assistance with MRI analysis. We acknowledge the Neuroergonomics Research Team of the University of Iowa's Department of Neurology for the use of their Grooved Pegboard normative data, obtained in their NIH-sponsored research.

\section{Author Contributions}

Conceived and designed the experiments: HSM TRB RGM. Performed the experiments: AJL BP. Analyzed the data: AJL BP ADM PMR TRB. Contributed reagents/materials/analysis tools: HSM TRB RGM. Wrote the paper: AJL BP RGM ADM PMR TRB HSM. 


\section{References}

1. Pantoni L (2010) Cerebral small vessel disease: from pathogenesis and clinical characteristics to therapeutic challenges. The Lancet Neurology 9: 689-701.

2. Zhou A, Jia J (2009) Different cognitive profiles between mild cognitive impairment due to cerebral small vessel disease and mild cognitive impairment of Alzheimer's disease origin. Journal of The International Neuropsychological Society 15: 898-905.

3. O'Sullivan M, Jones DK, Summers PE, Morris RG, Williams SC, et al. (2001) Evidence for cortical "disconnection" as a mechanism of age-related cognitive decline. Neurology 57: 632-638.

4. Junqué C, Pujol J, Vendrell P, Bruna O, Jódar M, et al. (1990) Leuko-araiosis on magnetic resonance imaging and speed of mental processing. Archives of Neurology 47: 151-156.

5. Sachdev PS, Brodaty H, Valenzuela MJ, Lorentz LM, Koschera A (2004) Progression of cognitive impairment in stroke patients. Neurology 63: 16181623.

6. Awad IA, Johnson PC, Spetzler RF, Hodak JA (1986) Incidental subcortical lesions identified on magnetic resonance imaging in the elderly. II. Postmortem pathological correlations. Stroke 17: 1090-1097.

7. O'Sullivan M, Morris RG, Huckstep B, Jones DK, Williams SCR, et al. (2004) Diffusion tensor MRI correlates with executive dysfunction in patients with ischaemic leukoaraiosis. Journal of Neurology, Neurosurgery and Psychiatry 75: 441-447.

8. Holtmannspötter M, Peters N, Opherk C, Martin D, Herzog J, et al. (2005) Diffusion magnetic resonance histograms as a surrogate marker and predictor of disease progression in CADASIL: a two-year follow-up study. Stroke 36: 25592565 .

9. Schmidt R, Ropele S, Ferro J, Madureira S, Verdelho A, et al. (2010) Diffusionweighted imaging and cognition in the leukoariosis and disability in the elderly study. Stroke 41: e402-e408.

10. Burzynska AZ, Preuschhof C, Backman L, Nyberg L, Li SC, et al. (2010) Agerelated differences in white matter microstructure: region-specific patterns of diffusivity. Neuroimage 49: 2104-2112.

11. Nitkunan A, Barrick TR, Charlton RA, Clark CA, Markus HS (2008) Multimodal MRI in cerebral small vessel disease: its relationship with cognition and sensitivity to change over time. Stroke 39: 1999-2005.

12. Nitkunan A, Lanfranconi S, Charlton RA, Barrick TR, Markus HS (2011) Brain atrophy and cerebral small vessel disease: a prospective follow-up study. Stroke 42: $133-138$.

13. Seo SW, Hwa Lee B, Kim EJ, Chin J, Sun Cho Y, et al. (2007) Clinical significance of microbleeds in subcortical vascular dementia. Stroke 38: 1949 1951.

14. Werring DJ, Frazer DW, Coward LJ, Losseff NA, Watt H, et al. (2004) Cognitive dysfunction in patients with cerebral microbleeds on $\mathrm{T}_{2}{ }^{*}$-weighted gradient-echo MRI. Brain 127: 2265-2275.

15. Patel B, Markus HS (2011) Magnetic resonance imaging in cerebral small vessel disease and its use as a surrogate disease marker. International Journal of Stroke 6: 47-59.

16. Bamford J, Sandercock P, Dennis M, Burn J, Warlow C (1991) Classification and natural history of clinically identifiable subtypes of cerebral infarction. Lancet 337: 1521-1526.

17. Fazekas F, Chawluk JB, Alavi A, Hurtig HI, Zimmerman RA (1987) MR signal abnormalities at $1.5 \mathrm{~T}$ in Alzheimer's dementia and normal aging. American Journal of Roentgenology 149: 351-356.

18. Charlton RA, Barrick TR, McIntyre DJ, Shen Y, O'Sullivan M, et al. (2006) White matter damage on diffusion tensor imaging correlates with age-related cognitive decline. Neurology 66: 217-222.

19. van Swieten JC, Koudstaal PJ, Visser MC, Schouten HJ, van Gijn J (1988) Interobserver agreement for the assessment of handicap in stroke patients. Stroke 19: 604-607.

20. Jenkinson M, Smith S (2001) A global optimisation method for robust affine registration of brain images. Medical Image Analysis 5: 143-156.

21. Neeman M, Freyer JP, Sillerud LO (1991) A simple method for obtaining crossterm-free images for diffusion anisotropy studies in NMR microimaging. Magnetic Resonance In Medicine 21: 138-143.
22. Grimaud J, Lai M, Thorpe J, Adeleine P, Wang L, et al. (1996) Quantification of MRI lesion load in multiple sclerosis: a comparison of three computer-assisted techniques. Magnetic Resonance Imaging 14: 495-505.

23. Cordonnier C, Potter GM, Jackson CA, Doubal F, Keir S, et al. (2009) improving interrater agreement about brain microbleeds: development of the Brain Observer MicroBleed Scale (BOMBS). Stroke 40: 94-99.

24. Smith SM, Zhang Y, Jenkinson M, Chen J, Matthews PM, et al. (2002) Accurate, robust, and automated longitudinal and cross-sectional brain change analysis. Neuroimage 17: 479-489.

25. Basser PJ, Mattiello J, LeBihan D (1994) Estimation of the effective self-diffusion tensor from the NMR spin echo. Journal of Magnetic Resonance Series B 103: 247-254.

26. Nelson H, Willison JR (1991) National Adult Reading Test (NART): Test Manual. NFER-Nelson, second edition.

27. Nagahama Y, Okina T, Suzuki N, Matsuzaki S, Yamauchi H, et al. (2003) Factor structure of a modified version of the wisconsin card sorting test: an analysis of executive deficit in Alzheimer's disease and mild cognitive impairment. Dementia and Geriatric Cognitive Disorders 16: 103-112.

28. R Development Core Team (2011) R: A Language and Environment for Statistical Computing. R Foundation for Statistical Computing, Vienna, Austria. http://www.R-project.org. ISBN 3-900051-07-0.

29. Charlton RA, Morris RG, Nitkunan A, Markus HS (2006) The cognitive profiles of CADASIL and sporadic small vessel disease. Neurology 66: 1523-1526.

30. Dormann CF, Elith J, Bacher S, Buchmann C, Carl G, et al. (2012) Collinearity: a review of methods to deal with it and a simulation study evaluating their performance. Ecography: In Press.

31. Smith EE, Schneider JA, Wardlaw JM, Greenberg SM (2012) Cerebral microinfarcts: the invisible lesions. The Lancet Neurology 11: 272-282.

32. Della Nave R, Foresti S, Pratesi A, Ginestroni A, Inzitari M, et al. (2007) Wholebrain histogram and voxel-based analyses of diffusion tensor imaging in patients with leukoaraiosis: correlation with motor and cognitive impairment. AJNR American Journal of Neuroradiology 28: 1313-1319.

33. Song SK, Sun SW, Ju WK, Lin SJ, Cross AH, et al. (2003) Diffusion tensor imaging detects and differentiates axon and myelin degeneration in mouse optic nerve after retinal ischemia. Neuroimage 20: 1714-1722.

34. Englund E (2002) Neuropathology of white matter lesions in vascular cognitive impairment. Cerebrovascular Diseases 13 Suppl 2: 11-15.

35. Viswanathan A, Godin O, Jouvent E, O'Sullivan M, Gschwendtner A, et al. (2010) Impact of MRI markers in subcortical vascular dementia: a multi-modal analysis in CADASIL. Neurobiology of Aging 31: 1629-1636.

36. Grassiot B, Desgranges B, Eustache F, Defer G (2009) Quantification and clinical relevance of brain atrophy in multiple sclerosis: a review. Journal of Neurology 256: 1397-1412.

37. Shiee N, Bazin PL, Zackowski KM, Farrell SK, Harrison DM, et al. (2012) Revisiting brain atrophy and its relationship to disability in multiple sclerosis. PLOS ONE 7: e37049.

38. Patel B, , Lawrence AJ, Chung A, Rich P, Mckinnon AM, et al. (In Press) Cerebral microbleeds and cognition in patients with symptomatic small vessel disease. Stroke.

39. Fisher CM (1968) The arterial lesions underlying lacunes. Acta Neuropathologica $12: 1-15$.

40. Khan U, Porteous L, Hassan A, Markus HS (2007) Risk factor profile of cerebral small vessel disease and its subtypes. Journal of Neurology, Neurosurgery and Psychiatry 78: 702-706.

41. Wheeler-Kingshott CAM, Cercignani M (2009) About "axial" and "radial" diffusivities. Magnetic Resonance In Medicine 61: 1255-1260.

42. O'Sullivan M (2010) Imaging small vessel disease: lesion topography, networks, and cognitive deficits investigated with MRI. Stroke 41: S154-S158.

43. Duering M, Zieren N, Hervé D, Jouvent E, Reyes S, et al. (2011) Strategic role of frontal white matter tracts in vascular cognitive impairment: a voxel-based lesion-symptom mapping study in CADASIL. Brain 134: 2366-2375.

44. Smith EE, Salat DH, Jeng J, McCreary CR, Fischl B, et al. (2011) Correlations between MRI white matter lesion location and executive function and episodic memory. Neurology 76: 1492-1499. 